\title{
Tapered Wire Antenna Design for Maximum Efficiency and Minimal Environmental Impact
}

\author{
Amir Galehdar ${ }^{1}$, David V. Thiel ${ }^{2}$, Steven G. O'Keefe ${ }^{3}$ \\ Centre for Wireless Monitoring and Applications, Griffith University \\ Nathan Qld 4111 Australia. \\ ${ }^{1}$ Amir.MohammadzadehGalehdar@student.griffith.edu.au \\ ${ }^{2}$ d.thiel@griffith.edu.au \\ ${ }^{3}$ s.okeefe@griffith.edu.au
}

\begin{abstract}
Antenna efficiency is an important parameter in RFID range and power consumption. Screen printed antennas often have poor conductivity and so increased loss. Designers offset this loss by increasing the width of the elements. This requires a substantial increase in the material required for construction. With billions of RFID antennas now in use worldwide, there is a need to reduce the environmental impact of the technology. A straight dipole antenna with decreasing width from the feed point to the ends achieved the same efficiency as a uniformly thick antenna. The design methodology is based on reducing the thickness of low current segments and increasing the thickness of high current segments.
\end{abstract}

\section{INTRODUCTION}

Some recent studies have aimed at decreasing the RFID tags cost by reducing the cost of production of the antenna [1]. One method of reducing cost is to minimize the antenna size. Most RFID tags are electrically small; i.e. $k r=0.1$ where $k$ is wave number and $r$ is the radius of a sphere enclosing the antenna. These antennas are often loaded with passive components to reduce the resonant frequency although this technique reduces the antenna efficiency and gain, which limits the reading range of the RFID system. Read range can be defined as the maximum distance at which the reader can detect the signal radiated from the tag [2]. Thus high cost and poor performance are the main factors that limit the implementation of the RFID on a greater scale.

This paper addresses these two issues - manufacturing cost and efficiency - simultaneously. The first section outlines the basic approach to changing the radius of wire antennas to reduce the amount of conducting material while maintaining performance. In the following sections, the concept is tested against a simple straight wire dipole antenna in the UHF band with $k r=1.64$.

\section{THEORY}

The behaviour of any excited piece of wire can be explained by Maxwell's equations which also limit the performance of small antennas [3]. Some antenna factors such as efficiency and gain can be improved within these rules. Antenna efficiency and gain are greatly affected by heating losses due to the finite conductivity of the conductor. This ohmic power loss depends on wire radius which can be equated to the width track of the printed antennas. Therefore changes in wire radius and conductivity affect the radiation efficiency and gain $[4,5]$.

\section{A. Efficiency}

While there are many methods used to calculate efficiency in an RFID antenna structure [6, 7], this paper presents efficiency results calculated from the method of moments calculation (NEC [8]). In this method the current is calculated in every segment, and the power loss is calculated in each segments of structure. The total power loss is summed and compared with input power (see [3] for further details of the method). If there are $N$ segments in the NEC model of the complete antenna and the wire segment length is small enough, the current over the length of the $i^{\text {th }}$ segment $i_{i}$, is approximately constant.

If $a_{i}$ is the radius of the $i^{\text {th }}$ segment, and all segment lengths are equal $\left(l_{i}=l=L / N\right)$ and the radius $\left(a_{i}=a\right)$ is unchanged throughout the structure, the power loss $P_{\text {loss }}$ can be written as:

$$
P_{\text {loss }}=\frac{1}{2} \sqrt{\frac{\pi f \mu_{0}}{\sigma}} \frac{l}{2 \pi a} \sum_{i=1}^{N} i_{i}^{2}
$$

where $f$ is the frequency, $\mu_{0}$ is the magnetic permeability, $L$ is the total length of the dipole, and $\sigma$ is the conductivity of the wire. The efficiency $\eta$ becomes:

$$
\eta=\frac{R_{i n} I^{2}-\sqrt{\frac{\pi f \mu_{0}}{\sigma}} \frac{l}{2 \pi a} \sum_{i=1}^{N} i_{i}^{2}}{R_{i n} I^{2}}
$$

where $R_{\text {in }}$ is the real part of the input impedance and $I$ is the current at the feed point. An increase in $a$ causes a reduction in ohmic loss in all segments and $\eta$ increases. While this relationship appears simple, a change in wire radius in one or more segments will change the current distribution. It can be seen in (2) that ohmic loss is large in those segments with larger current.

\section{B. Gain}

The power gain or simply the gain, $G_{p}$, of an antenna is defined as the ratio of the maximum radiation intensity to the radiation intensity of a lossless isotropic source the same power input [9]. This is related to $\eta$ by the equation: 


$$
\eta=\frac{G_{P}}{D}
$$

where $D$ is directivity. Based on these equations one can determine the gain of the antenna by noting that:

$$
P_{r}=P_{i n}-P_{\text {loss }} \Rightarrow G_{P}=D \frac{P_{r}}{P_{\text {in }}}
$$

where $P_{i n}$ is the power delivered to the antenna. Changes to the gain due to changes in wire radius affect the reading range in all RFID tags.

If the electromagnetic characteristics of materials near or in contact with tag are ignored, the reading range $R$ of a RFID tag can be estimated from the Friis free-space formula [3].

$$
R=\frac{\lambda}{4 \pi} \sqrt{\frac{P_{t} G_{t} G_{P} \tau}{P_{t h}}}
$$

where $\lambda$ is the free space wavelength, $P_{t}$ is the power transmitted by the reader, $G_{t}$ is the gain of the transmitting antenna, $G_{P}$ is the gain of the receiving tag antenna, $P_{t h}$ is the minimum threshold power necessary to provide enough power to the RFID tag chip, and $\tau$ is the power transmission coefficient given by:

$$
\tau=\frac{4 R_{c} R_{a}}{\left|Z_{c}+Z_{a}\right|^{2}}, \quad 0 \leq \tau \leq 1
$$

The output impedance of the active electronics $Z_{c}=R_{c}+j X_{c}$ and the antenna input impedance $Z_{a}=R_{a}+j X_{a}$ both feature in the denominator of equation (6)

\section{C. $\quad$ Tapering method}

A current distribution along a straight wire antenna near its resonant frequency approximates a sinusoidal. For example, a $166 \mathrm{~mm}$ long (i.e. $L=166 \mathrm{~mm}$ ), centre fed, dipole antenna with a copper wire radius of $0.05 \mathrm{~mm}$ resonates at $869 \mathrm{MHz}$. The wire structure was divided into $N=167$ equally sized segments and fed by a voltage source $(1 \mathrm{~V})$ at $87^{\text {th }}$ segment. The current in each segment was calculated using NEC and is plotted in Fig. 1.

The segments at or close to the feed ( $87^{\text {th }}$ segment) have the highest current. The extremities have the smallest currents. The loss is therefore higher in those segments close to the feed.

The efficiency and gain increases when the wire radius is increased. By tapering the radius of wire based on current distribution, the wire radius in high current areas was selectively increased. The power loss was reduced and the efficiency and gain increased. However other antenna performance characteristics also changed. For example, the resonant frequency varied and this was corrected by changing the over-all antenna length. Wire radius changes were investigated for a straight wire dipole and results are presented in detail in the following sections.

Two different methods of tapering were applied.

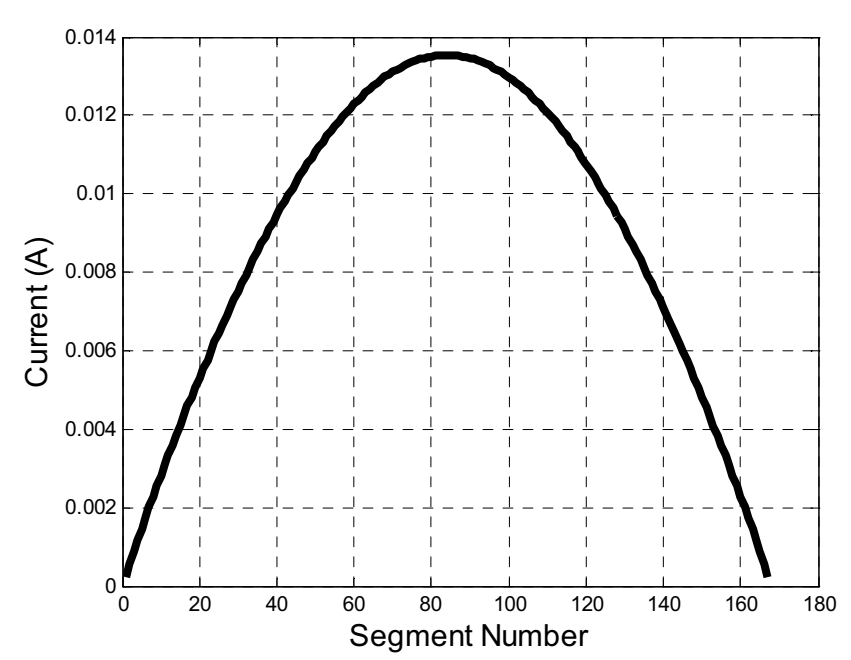

Fig. 1: Current distribution of 166mm dipole antenna with 167 segments.

(a) Method 1: The wire radius at the feed point $a_{\text {feed }}$ was fixed and the radius at every other segment $a_{i}$ was decreased proportionally to the original current distribution $i_{i}$. Following equation (1), the obvious choice would be to scale the radius proportionally to the current squared, this results in a very wide range of segment radius and the antenna is almost impossible to construct physically. Thus $a_{i}$ was calculated using a linear current scaling factor from:

$$
a_{i}=\left\{\begin{array}{lll}
a_{\text {feed }} \frac{i_{i}}{i_{\text {feed }}} & \text { for } & a_{i} \geq 0.001 \mathrm{~mm} \\
0.001 \mathrm{~mm} & \text { for } & a_{i} \leq 0.001 \mathrm{~mm}
\end{array}\right.
$$

The $0.001 \mathrm{~mm}$ limit was chosen to prevent NEC error. It was found that most antenna characteristics remained virtually unchanged even though the amount of conductive material required, and so the total cost of the antenna was significantly reduced.

(b) Method 2: An iterative approach was adopted to ensure a linear relationship between current and wire radius. Each segment radius $a_{i}$ was changed interactively as the current in the segment $i_{i}$ changed with every new configuration. A further restriction was that the overall copper volume was fixed. This required rescaling the radius of every segment of the antenna after each iteration. The original copper volume $V_{0}$ was calculated from:

$$
V_{0}=2 \pi a^{2} L
$$

where $a$ is the radius of the wire and $L$ is the total length. The volume of the tapered antenna $V_{T}$ was calculated using:

$$
V_{T}=\sum_{i=1}^{N} 2 \pi a_{i}^{2} l_{i}
$$

where $l_{i}$ is the length of the $i^{\text {th }}$ segment and $a_{i}$ is calculated from (7). The segment current $i_{i}$ was changed when $a_{i}$ changed. The final copper distribution was obtained using the algorithm shown in Fig. 2. The iterative scheme was found to converge within a fixed radius tolerance after approximately 150 iterations using a radius change of less than $0.001 \mathrm{~mm}$ as the 
termination criterion. In this method, the cost of materials is unchanged, but the antenna performance is enhanced.

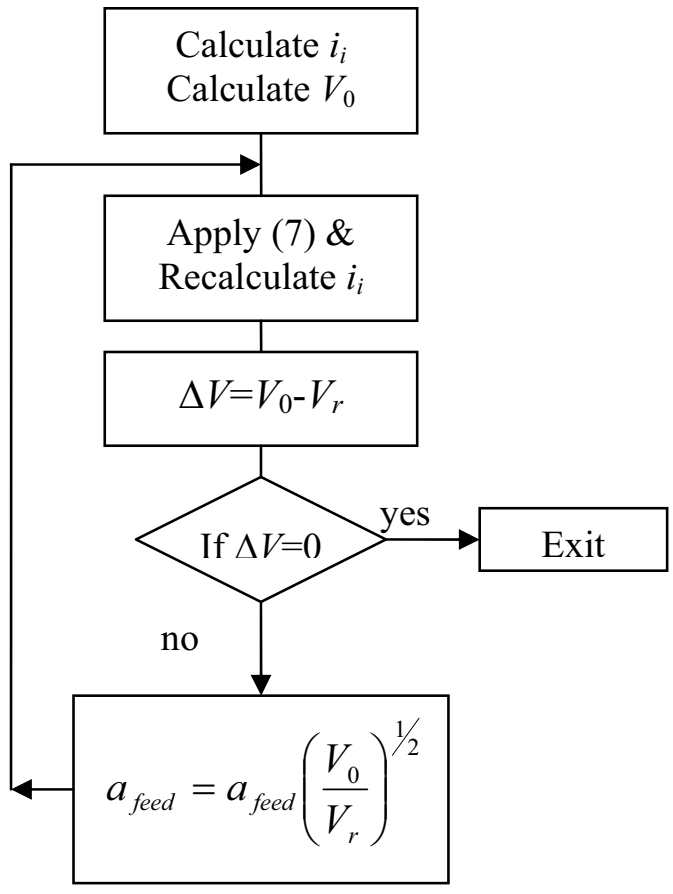

Fig. 2: Method 2 radius taper algorithm with fixed volume $V_{0}$

\section{RESULTS}

\section{A. Uniform Radius}

A dipole antenna (length $166 \mathrm{~mm}$, radius $0.05 \mathrm{~mm}$, centre fed) resonates at $869 \mathrm{MHz}$ (Europe UHF RFID band). The wire radius was changed stepwise from 0.025 to $0.1 \mathrm{~mm}$ and the effect on both resonant frequency $f_{0}$ and efficiency $\eta$ was noted. NEC results were calculated using 167 equally sized segments and the efficiencies calculated using (2). The length of antenna was unchanged. The efficiency increased and the resonant frequency decreased with radius (see Fig. 3). The modelling was conducted over the frequency range $870 \mathrm{MHz}$ to $900 \mathrm{MHz}$ in $1 \mathrm{MHz}$ steps and so the variations in resonant frequency are given as discrete $1 \mathrm{MHz}$ steps in Fig. 3.

Fig. 4 shows the variation in antenna gain and ohmic power loss. As expected, the gain increased and the power loss decreased as the radius was increased. The decrease in ohmic power loss corresponds to the reduction in wire resistance.

\section{B. Non-Uniform Radius}

In Method 1, the radius at the feed point was fixed and the radius of all other segments was decreased proportionally based on the original current distribution. Most antenna characteristics remained unchanged, however, the resonant frequency increased in both cases. In Method 2, the copper volume was kept constant and the wire radius varied stepwise with the current distribution. This resulted in an antenna with the same shape as that from Method 1, but slightly wider closer to the feed (see Fig. 5). The gain, efficiency and resonant frequency all increased when compared to the original dipole. There is no significant difference in antenna performance between the antennas designed using the two methods, however, Method 1 uses 50\% less copper compared to the original dipole. The results from the two tapering methods are summarized in Table 1 . The radiation performance of all three antennas is very similar and $k r \approx 1.6$ for all three cases.

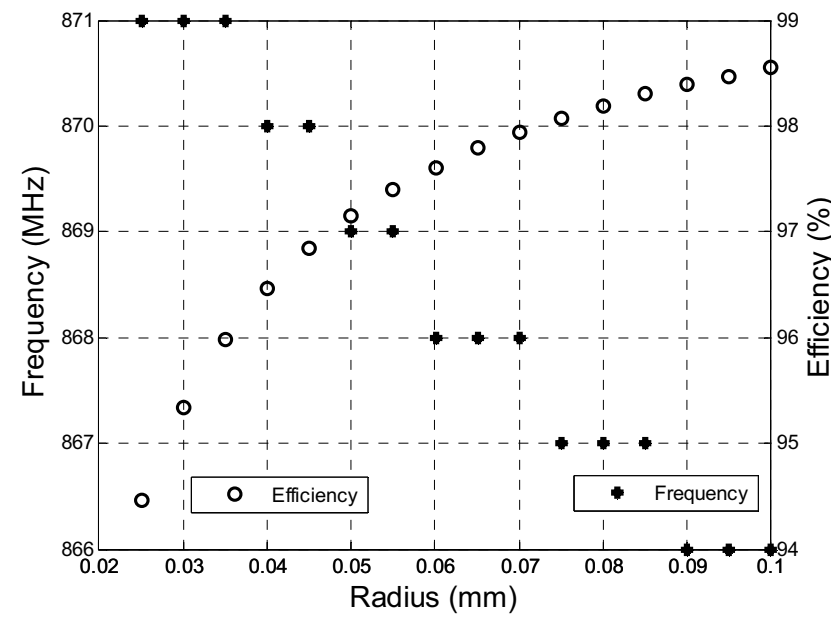

Fig. 3: Dipole efficiency and resonant frequency results for a uniform straight wire dipole with varying radius.

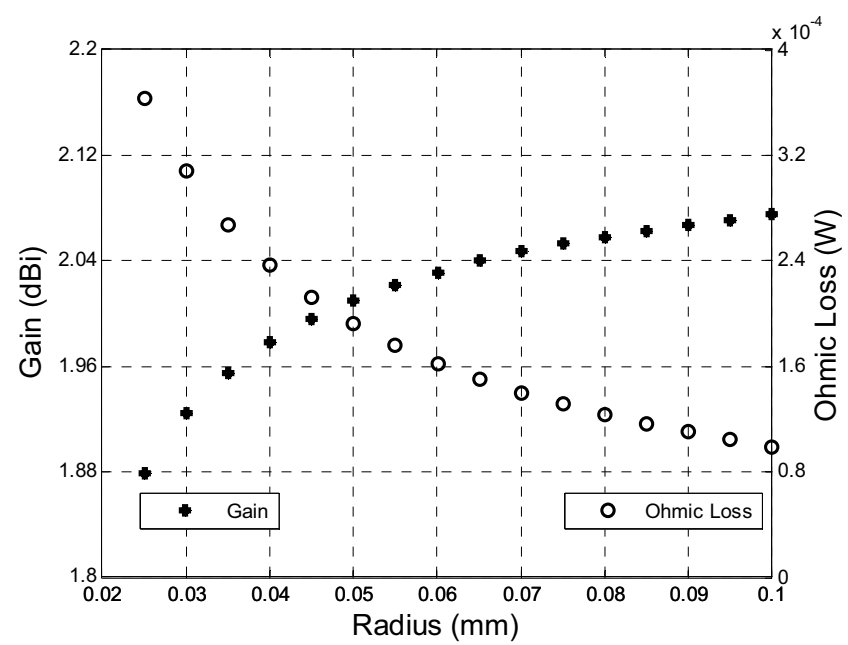

Fig. 4: Dipole gain and ohmic power loss for a uniform, straight wire dipole with varying wire radius.

TABLE I

ANTENNA CHARACTERISTICS FOR TWO TAPERING STRATEGIES,

\begin{tabular}{|l|c|c|c|c|c|c|}
\hline & $\begin{array}{c}\boldsymbol{a}_{\text {feed }} \\
\mathrm{mm}\end{array}$ & $\begin{array}{c}\boldsymbol{\eta} \\
(\%)\end{array}$ & $\begin{array}{c}\boldsymbol{f}_{\mathbf{0}} \\
\mathrm{MHz}\end{array}$ & $\begin{array}{c}\boldsymbol{R}_{\text {in }} \\
\Omega\end{array}$ & $\begin{array}{c}\boldsymbol{V}_{\mathbf{0}} \\
\mathrm{mm}^{3}\end{array}$ & $\begin{array}{c}\text { Gain } \\
\mathrm{dB}\end{array}$ \\
\hline Uniform & 0.05 & 97.1 & 869 & 74.0 & 1.3 & 2.10 \\
\hline Method 1 & 0.05 & 96.5 & 899 & 73.9 & 0.67 & 2.10 \\
\hline Method 2 & 0.07 & 97.4 & 898 & 73.2 & 1.3 & 2.16 \\
\hline
\end{tabular}




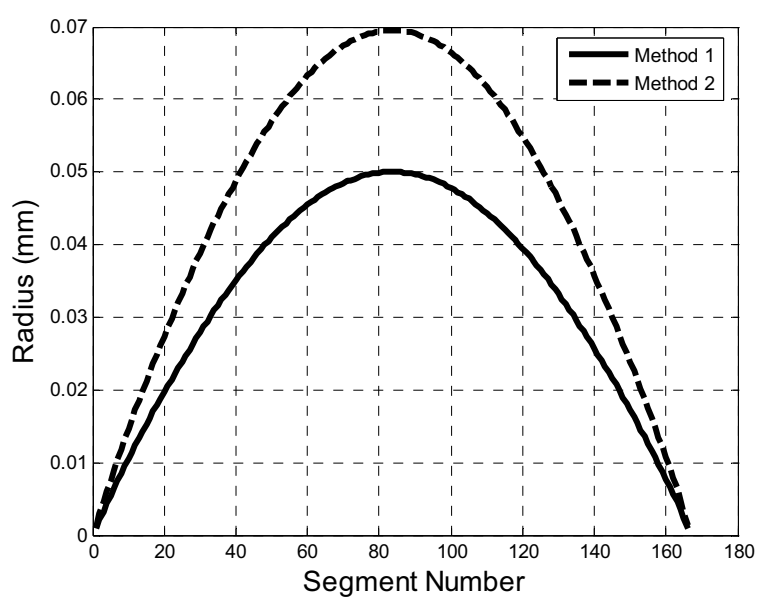

Fig. 5: Change in wire radius for the two tapering methods.

\section{CONCLUSION}

In the past many antenna engineers have not paid significant attention to the environmental cost of their structures. This feature is not particularly important when manufacturing volume is low and the energy requirements in manufacture did not present a significant cost. Recently there has been considerable emphasis on full life-cycle costing of products. The advent of high volume production of antennas in such markets such as mobile telephones and RFID tags, these issues become particularly important and must be addressed.

This paper has addressed two significant short-comings of RFID tags which inhibit their use: expense and performance. These two problems were tackled by introducing a novel method of tapering the wire radius of antennas. It was shown that by increasing the wire radius, the loss in the structure reduces, and as a result the efficiency and gain are improved. However, it is seen that in the dipole, the bigger the wire radius, the more resonant frequency decreases and results were explained in terms of increasing the capacitance effect of the end of antenna. Based on these results a new tapering method was introduced. Two methods were investigated: firstly by reducing the material needed to produce antenna, and secondly based on enhancing the performance by using the same amount of material as original antenna. These two methods were used to design RFID antennas with better performance and/or reduced manufacturing costs for a tag with a fixed area.

Further work with these tapering algorithms applied to the more traditional meander line RFID antennas will be reported in the near future.

\section{REFERENCES}

[1] S. V. Subramanian, J. M. J. Frechet, P. C. Chang, D. C. Huang, J. B Lee. A. R. Murphy, D. R. Redinger and S. K. Volkman, "Progress Toward Development of All-Printed RFID Tags: Materials, Process, and Devices," IEEE Proc. vol. 93, pp.1330-1338, July 2005.

[2] J. G. Marrocco, "Gain-optimized self-resonant meander line antenna for RFID application," IEEE Trans. Antenna Wireless Propag. Lett. vol. 2, pp. 302-305, 2003

[3] A. Galehdar, D. V. Thiel and S. G. O'Keefe, “Antenna Efficiency Calculations for Electrically small, RFID antennas," IEEE Antenna and Wireless Propag., vol.6, pp. 156-159, 2007.

[4] H. A. Wheeler, "Fundamental limitation of small antenna," Proceeding of the IRE, pp.1479-1484, Dec. 1947.

[5] S. R. Best, "A Discussion on the Properties of Electrically Small SelfResonant Wire Antennas," IEEE Antennas Propag. Mag. vol.46, pp. 922, 2004.

[6] E. H. Newman, P. Bohley, and C. H. Walter, "Two methods for the measurement of antenna efficiency," IEEE Trans. Antennas Propag., vol. 23, pp. 457-461, 1975.

[7] W. E. McKinzie, "A modified Wheeler Cap method for measuring antenna efficiency," in Proc. IEEE AP-S Int. Symp. Dig., vol. 1, Issue 13-18, Jul. 1997, pp. 542-545.

[8] NEC-Win Plus, User's Manual, Nittany Scientific Inc, 2003.

[9] D. K. Cheng, Field and wave electromagnetics, 2nd ed., Addison Wesley, 1989 